\title{
MULTI-MODEL CONTROL OF A SIMULATED PH NEUTRALIZATION PROCESS
}

\author{
Jari M. Böling *,1 Dale E. Seborg** \\ João P. Hespanha ${ }^{* *, 2}$ \\ * Process Control Laboratory, Åbo Akademi University, \\ Finland \\ ** Department of Chemical Engineering, University of \\ California, Santa Barbara \\ *** Department of Electrical and Computer Engineering, \\ University of California, Santa Barbara
}

\begin{abstract}
A multi-model PID controller is developed and evaluated in a simulation study for a nonlinear $\mathrm{pH}$ neutralization process. The performance and robustness characteristics of the multi-model controller are compared to those for conventional PID controllers and an alternative "multi-model interpolation" controller.

Keywords: Adaptive control, switching control, multiple models, PID control
\end{abstract}

\section{INTRODUCTION}

Many industrial processes inevitably change over time for a variety of reasons that include: equipment changes, different operating conditions, or changing economic conditions. Consequently, a fundamental control problem is how to provide effective control of complex processes where significant process changes can occur, but cannot be measured or anticipated. The conventional solution is conservative controller tuning for worst case conditions. However, this approach can result in poor control system performance for more typical conditions. Alternatively, adaptive control strategies are available where the controller parameters and/or control structure are modified on-line as conditions change (Åström and Wittenmark, 1995).

This paper is concerned with a special class of adaptive control strategies referred to as switching control or multi-model control (Johansen and Murray-Smith, 1997; Hespanha, 2001). The motivation for multi-model control is that for many complex technical processes, the local behavior can be captured at least approximately by a set of relatively simple models. Also, a corresponding feedback controller can be designed for each

\footnotetext{
1 Supported by the research institute of the foundation of Åbo Akademi University, and by the Academy of Finland under grant number 203819.

2 Supported by NSF Grant \#ECS-0242798.
}

individual model. For these situations, an adaptive control approach based on selecting the best model (and controller) for the current conditions provides a promising approach. Selection of the performance criterion and switching strategy is a key design issue.

\section{MULTI-MODEL PID CONTROL}

In multi-model control, a bank of candidate models (and/or controllers) are specified a priori. Then a supervisory controller selects the most appropriate model (or controller) for the current conditions. For each model, a suitable controller can be designed off-line. The on-line controller switching is based on the performance evaluation of the bank of models (and/or controllers). Control problems involving transitions between known operating regimes are readily handled by a multi-model approach (Johansen and MurraySmith, 1997). Multi-model control is also applicable to more general control problems where operating regimes cannot be determined a priori (Narendra and Balakrishnan, 1997; Hespanha, 2001). For example, the capabilities of multimodel control have been successfully demonstrated for drug infusion control where variability and unpredictability are key issues (Schott and Bequette, 1997).

In this paper, we consider a multi-model strategy for PID controllers that is based on a set of 
simple linear dynamic models. Each model has the same structure but different values of the model parameters. Grids of parameter values are assigned based on an assumed range for each model parameter. The ranges can be determined from a priori knowledge of expected operating conditions. For example, ranges for process gains and time constants can be specified based on physical knowledge such as the maximum and minimum values of temperatures and product flow rate. The grid spacing does not have to be constant.

In Section 3 the multi-model strategy is compared to a novel adaptive control strategy where the controller is automatically re-tuned after poor performance is detected (Wojsznis and Blevins, 2002). The re-tuning is based on re-estimating model parameters from recent input/output data.

A block diagram for the multi-model control strategy considered in this paper is shown in Figure 1, where $u$ is the input, $y$ is the output, $d$ is the unmeasured disturbance, and $y_{s p}$ is the setpoint.

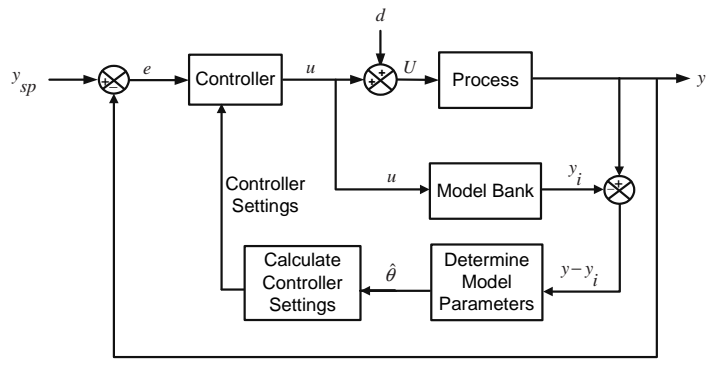

Fig. 1. Block diagram of multi-model control.

The model parameters $\hat{\theta}$ for the current conditions are determined by calculating a performance index for each model. For example, the low-pass filtered squared prediction error for each model, $\pi_{i}$, can be calculated,

$$
\pi_{i}(k)=\lambda \pi_{i}(k-1)+(1-\lambda) \varepsilon_{i}^{2}(k)
$$

where $\varepsilon_{i}(k)$ denotes the one-step prediction error for model $i$ at time $k$. The filter constant $\lambda$ can be interpreted as a forgetting factor, as will be discussed later.

At each time $k$, the performance index of the currently chosen model, $\pi_{c}(k)$, is compared with the values for the other models. If

$$
\pi_{c}(k)>(1+h) \min _{i} \pi_{i}(k)
$$

the model with the smallest value of $\pi$ is selected and the corresponding controller is implemented. In Eq. (2), $h>0$ is a hysteresis parameter that prevents excessive switching. Both in theory and in practice, it is important that excessive switching be avoided. The use of a hysteresis term is a convenient approach for fulfilling this requirement.

\subsection{Process model and unmeasured disturbances}

Unmeasured disturbances can be a significant problem for adaptive control strategies, including multi-model control. For example, additive disturbances can result in adaptation of model parameters when the parameters have not actually changed. The resulting incorrect model parameters can produce very poor control. For multimodel control applications, the unknown disturbance can be approximated as a bias term for either the input or the output. Consequently, the bank of models can include different disturbance magnitudes, as well as different values of the other model parameters.

In this paper, the multi-model control strategy is based on a single-input, single-output model, namely, a first-order plus time-delay model with an additive input disturbance:

$$
\begin{array}{r}
y_{i}(k+1)=a_{i} y(k)+b_{i}\left(u\left(k-\ell_{i}\right)+d_{i}\right), \\
i=1, . ., M
\end{array}
$$

The model parameters are $a_{i}, b_{i}, d_{i}$ and time delay $\ell_{i}$, where subscript $i$ denotes the model index. For the simulation examples in Section 3, the parameters $a_{i}$ and $\ell_{i}$ are assumed to be known. Thus, the model bank consists of models in the form of (3) with different values of $b_{i}$ and $d_{i}$. When an unmeasured disturbance occurs, it is mapped to an approximately equivalent disturbance $d_{i}$ in the model bank. Although the disturbance estimate is only a rough approximation of the actual disturbance, it can result in excellent switching control as demonstrated by the simulations in Section 3 .

For disturbance $d_{i}$, a constant grid spacing is reasonable. However, for the gain parameter $b_{i}$, it is more appropriate to use a logarithmically spaced grid.

\subsection{Forgetting of past data}

In adaptive control applications, past data must be discounted (i.e., forgotten) in order to have the adaptive controller to respond in a timely manner to process changes. Typically, a forgetting factor is employed such as $\lambda$ in (1), where $0 \leq \lambda \leq 1$. The specification of $\lambda$ involves an inherent tradeoff. If $\lambda$ is too large, the adaptation is too sluggish while if $\lambda$ is too small, the adaptation is overly aggressive resulting in loss of relevant information and excessive adaptation. In this paper, an ad hoc forgetting of past data is employed. The basic premise is that when there is little input excitation, forgetting of past data is suspended by setting $\lambda=1$. On the other hand, when there is sufficient excitation, $\lambda$ is set equal to a specified constant, $\lambda_{0}$. Two metrics are considered as measures of the degree of process excitation: 
(1) The prediction error, $\varepsilon=y-y_{c}$.

(2) The control error, $e=y_{s p}-y$.

During a period where the metric exceeds a specified threshold, $\lambda$ is set equal to $\lambda_{0}<1$. Otherwise, it has the nominal value of one and no forgetting of past data occurs.

Both of these alternatives are evaluated in Section 3. The threshold for each metric was chosen to be ten times larger than the expected value for normal operating conditions.

\subsection{Controller design}

The PID controller design included a low pass filter of the error signal:

$$
G_{c}(s)=K_{c}\left(1+\frac{1}{\tau_{I} s}+\tau_{D} s\right)\left(\frac{1}{\tau_{f} s+1}\right)
$$

The controller was designed using a first-orderplus-time-delay (FOPTD) model that corresponds to (3) with no disturbance:

$$
\frac{y(s)}{u(s)}=\frac{K e^{-L s}}{\tau s+1}
$$

The controller parameters were calculated using an IMC design procedure (Morari and Zafiriou, 1989) and implemented as the equivalent discretetime version.

\section{SIMULATIONS}

In this section, the proposed switching PID controller (SPID) is evaluated in simulation studies for two examples: (i) a physical nonlinear model of the UCSB pH neutralization process (Hall and Seborg, 1989), and (ii) an approximate FOPTD model of the process. The stirred tank neutralizer has three dilute inlet streams: base $(\mathrm{NaOH})$, acid $\left(\mathrm{HNO}_{3}\right)$, and buffer $\left(\mathrm{NaHCO}_{3}\right)$. The exit stream $\mathrm{pH}$ is controlled by adjusting base flow rate $Q_{3}$ while the liquid level is regulated by manipulating acid flow rate $Q_{1}$. The buffer flow rate $Q_{2}$ is the major unmeasured disturbance. The $\mathrm{pH}$ neutralization process is highly nonlinear as indicated by the static map shown in Fig. 2.

The linear model serves as a simple approximate model for the nonlinear $\mathrm{pH}$ process. This model is in the form of Eq. (5) and the nominal parameter values are $K=1, \tau=3.6 \mathrm{~min}$, and $L=0.75$ min. For the $\mathrm{pH}$ process, only the process gain $K$ varies significantly for the normal range of operating conditions. Thus, in order to keep the simulation relatively simple and illustrative, the time constant $\tau$ and the delay $L$ were assumed to be constant. Only the process gain $K$ and the assumed input disturbance $d$ were varied.

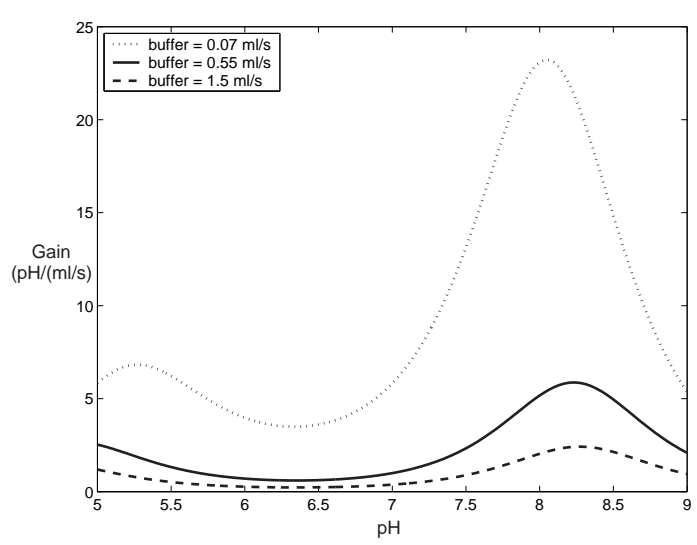

Fig. 2. $\mathrm{pH}$ gain variation.

The parameter ranges and grid spacing of the parameters were based on a reasonable range of operating conditions for the physical process. The gains in the model bank varied between 0.3 and 22 , with ten logarithmically-spaced values of the gain, both above and below the nominal value of one. For the input disturbances in the model bank, 59 equally-spaced values were assumed for the interval between -2.9 and +2.9 . Thus, the model set consisted of a total of 1239 models for each simulation. However, similar results were obtained for model banks with much smaller numbers of models. The hysteresis parameter $h$ in Eq. (2) was set equal to 1 .

In the simulation studies, five PID control strategies were evaluated:

(1) A nominal controller designed for the nominal model in (5) with IMC parameter, $\tau_{c}=$ $1.5 \mathrm{~min}$.

(2) A conservatively-tuned controller designed for a "high gain condition" of $K=3.5$. This controller was designed using $\tau_{c}=0.75 \mathrm{~min}$.

(3) A multi-model (switching) controller with the forgetting factor based on the prediction error.

(4) A multi-model (switching) controller with the forgetting factor based on the control error.

(5) An adaptive controller based on "multiple model interpolation (MMI)" and intermittent controller re-tuning.

In the MMI adaptive control strategy developed by Emerson Process Management (Wojsznis and Blevins, 2002), after poor controller performance is detected, data are collected for a specified period of time (e.g., the open-loop settling time). Then the model parameters are re-estimated and the corresponding model-based controller is updated. Various criteria can be used to detect poor controller performance. In this application, data collection is initiated when the control error exceeds 100 times the expected value for nominal conditions. Then data were collected for a period 
of 10 min. The FOPTD model and IMC controller design for the SPID approach were also used for the re-tuning method. However, an output disturbance, rather than an input disturbance, was assumed.

Initially, the five controllers were evaluated for the linear system in Eq. (5). The results are summarized in Figs. 3 through 5 and Table 1. The controllers were compared for a setpoint change at $t=5 \mathrm{~min}$, followed by a step disturbance at $t=40 \mathrm{~min}$. For the high gain model $(\mathrm{K}=3.5)$, the nominal controller produce the very oscillatory response in Fig. 3. However, the switching controllers in Figs. 4 and 5 readily adapt to the changing conditions without excessive oscillations. The forgetting factor and estimated model parameters are also shown in these two figures.
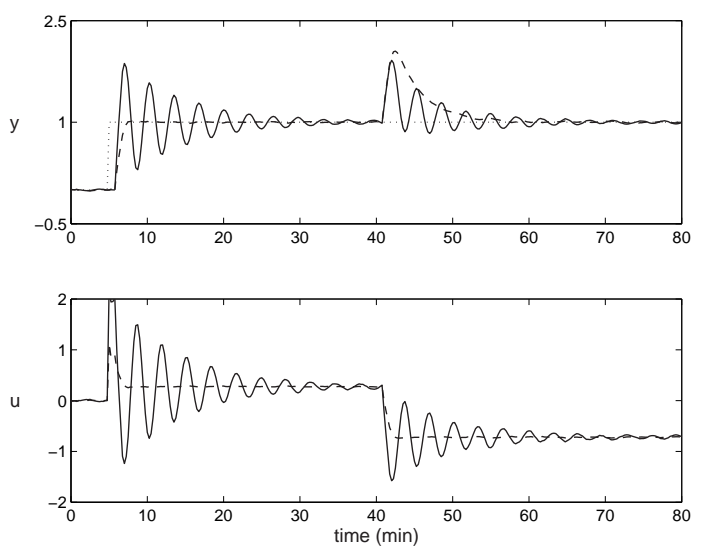

Fig. 3. Control of high-gain linear model: Nominal controller $(-)$, conservative controller (- - -), and setpoint $(\cdots)$.

\subsection{The nonlinear $p H$ simulation}

The five controllers were further evaluated by having the nonlinear $\mathrm{pH}$ model serve as the "process". Initially, the controllers were tested for a series of three step changes in the buffer flow rate $Q_{2}$ : a decrease from 0.55 to $0.07 \mathrm{ml} / \mathrm{s}$ at $t=5 \mathrm{~min}$, then an increase to $1.5 \mathrm{ml} / \mathrm{s}$ at $t=30 \mathrm{~min}$, and finally a return to the initial value at $t=75 \mathrm{~min}$. Note that the process gain increases as $Q_{2}$ decreases, as shown in Fig. 2.

Figure 6 indicates that the nominal PID controller resulted in an unstable response after the first $Q_{2}$ disturbance, while the conservatively-tuned controller is stable but rather sluggish for the other two disturbances. The two switching controllers in Figs. 7 and 8 provided satisfactory control for all three disturbances. Their performance is about the same, regardless of whether the control error or the prediction error is used to specify the forgetting factor.
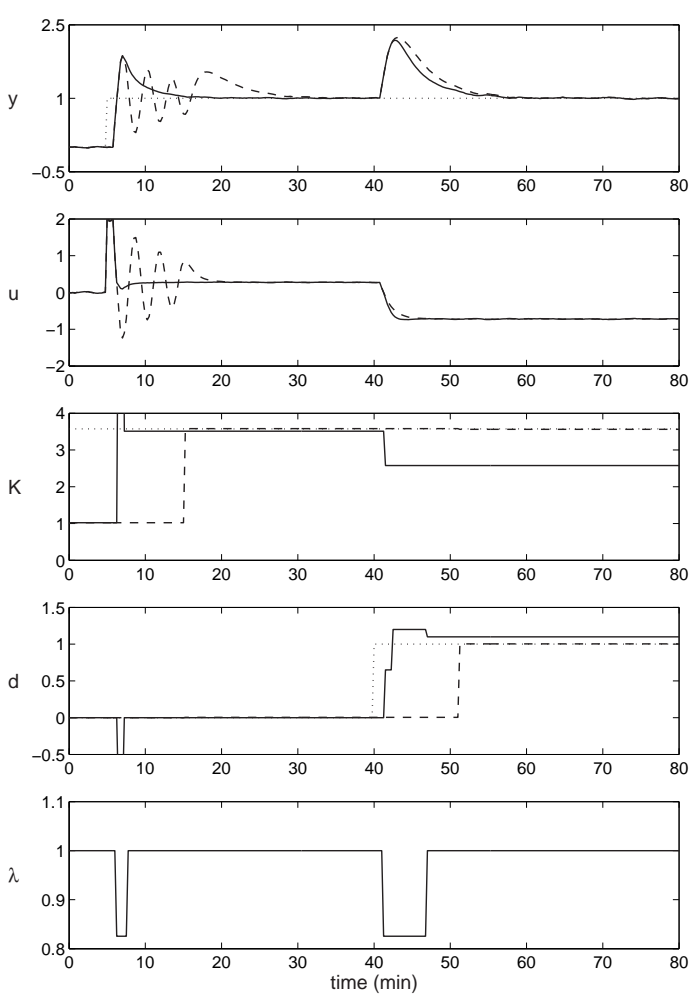

Fig. 4. Control of high-gain linear model: Switching controller (-), re-tuning controller (- $-)$, setpoint and true values $(\cdots)$. Prediction error used in forgetting.

Since the re-tuning adaptive controller was initialized with the nominal controller setting, its initial response to the first disturbance is also oscillatory. However, after the ten minute data collection period finishes at $t=20 \mathrm{~min}$, the retuned controller is quite satisfactory. Note that the "true values" of gain $K$ and disturbance $d$ in Fig. 6 and subsequent figures are local values for the current value of $Q_{2}$. Similar results were obtained for the setpoint changes in Figs. 9 and 10. The process gain changed by over a factor of seven during these setpoint changes, as is apparent from Fig. 2.

Finally, the values of the Integral Absolute Error performance index for both simulation examples are reported in Table 1, with the best values shown in boxes.

\section{CONCLUSIONS}

A multi-model PID control strategy has been evaluated in two simulation studies that included comparisons with three other PID controllers: a re-tuning adaptive controller and two nonadaptive controllers. The simulations indicated that the multi-model controller was quite effective over wide ranges of unmeasured disturbances and process changes. The re-tuning strategy also performed very well, but was slower to respond to sudden disturbances. 
Table 1. The Integral Absolute Errors for the simulations.

\begin{tabular}{|c|c|c|c|c|c|}
\hline \multicolumn{6}{|c|}{ Nominal linear system } \\
\hline & n-PID & c-PID & $\varepsilon$-sw. & $e$-sw. & re-tune \\
\hline setpoint & 9.2 & 20.4 & 9.2 & 9.2 & 9.2 \\
\hline disturb. & 9.6 & 21.0 & 9.0 & 8.3 & 9.5 \\
\hline total & 18.8 & 41.4 & 18.2 & $\mathbf{1 7 . 5}$ & 18.7 \\
\hline \multicolumn{6}{|c|}{ High-gain linear system } \\
\hline & n-PID & c-PID & $\varepsilon$-sw. & $e$-sw. & re-tune \\
\hline setpoint & 24.7 & 6.6 & 14.9 & 22.7 & 32.1 \\
\hline disturb. & 15.1 & 21.3 & 25.8 & 10.5 & 32.5 \\
\hline total & 39.8 & 27.9 & 40.7 & 33.2 & 64.6 \\
\hline
\end{tabular}

\begin{tabular}{|l|ccccc|}
\hline \multicolumn{7}{|c|}{$p H$ system } \\
\cline { 1 - 5 } & n-PID & c-PID & $\varepsilon$-sw. & $e$-sw. & re-tune \\
\hline$Q_{2} \quad 0.55 \rightarrow .07$ & $81.4^{*}$ & 14.8 & 8.5 & 11.8 & 19.2 \\
$Q_{2} \quad 0.07 \rightarrow 1.5$ & $25.3^{* *}$ & 41.7 & 32.7 & 16.4 & 42.3 \\
$Q_{2} \quad 1.5 \rightarrow 0.55$ & 12.5 & 26.5 & 12.7 & 4.6 & 12.1 \\
\hline total buffer & $\mathbf{1 1 9 . 2}$ & $\mathbf{8 3 . 0}$ & $\mathbf{5 3 . 9}$ & $\mathbf{3 2 . 8}$ & $\mathbf{7 3 . 6}$ \\
\hline setpoint $7 \rightarrow 8$ & $40.7^{*}$ & 13.5 & 19.8 & 16.3 & 31.5 \\
setpoint $8 \rightarrow 6$ & $19.2^{* *}$ & 40.7 & 44.5 & 35.7 & 46.3 \\
setpoint $6 \rightarrow 7$ & 13.7 & 30.4 & 12.7 & 10.3 & 13.3 \\
\hline total setpoint & $\mathbf{7 3 . 6}$ & $\mathbf{8 4 . 6}$ & $\mathbf{7 7 . 0}$ & $\mathbf{6 2 . 3}$ & $\mathbf{9 1 . 1}$ \\
\hline total pH & $\mathbf{1 9 2 . 8}$ & $\mathbf{1 6 7 . 6}$ & $\mathbf{1 3 0 . 9}$ & $\mathbf{9 5 . 1}$ & $\mathbf{1 6 4 . 7}$ \\
\hline
\end{tabular}
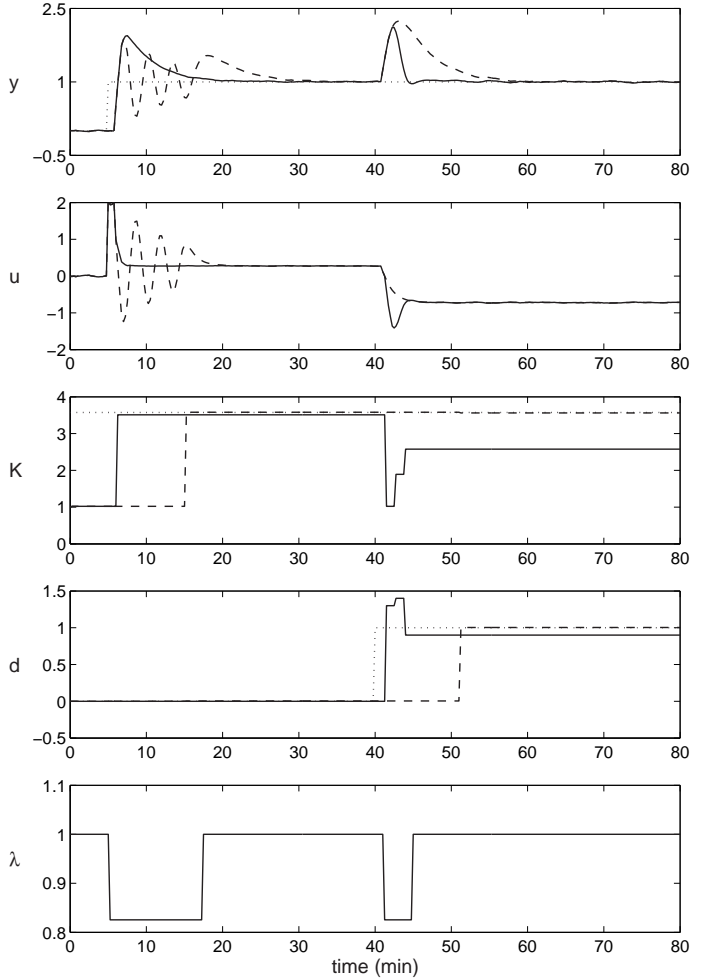

Fig. 5. Control of high-gain linear model: Switching controller $(-)$, re-tuning controller (- - ), setpoint and true values $(\cdots)$. Control error used in forgetting.

\section{REFERENCES}

Åström, K.J. and B. Wittenmark (1995). Adaptive Control. 2nd ed.. Addison Wesley, NY.

Hall, R. C. and D. E. Seborg (1989). Modelling and self-tuning control of a multivariable $\mathrm{pH}$ neutralization process, part I: Modelling and multiloop control. In: Proc. ACC. Pittsburgh, PA. pp. 1822-1828.

Hespanha, J. P. (2001). Tutorial on supervisory control. Lecture Notes for the workshop Control Using Logic and Switching for the 40th IEEE CDC, Orlando, FL. Available at http://www.ece.ucsb.edu/ hespanha/ techreps.html.

Johansen, T. A. and R. Murray-Smith (1997). The operating regime approach. In: Multiple
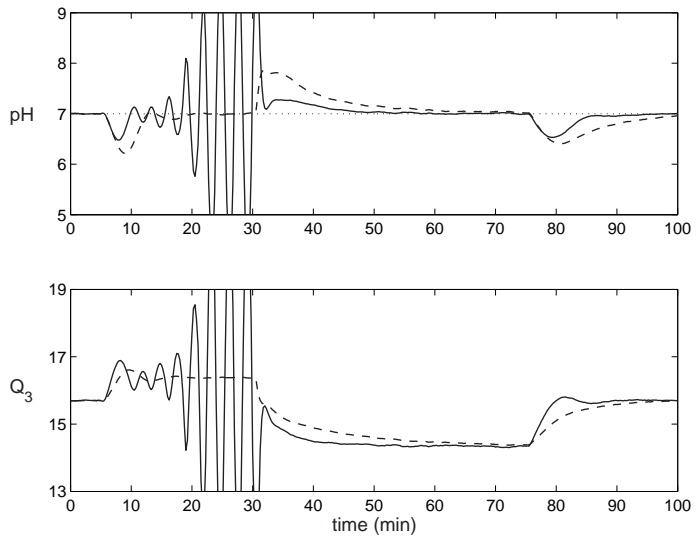

Fig. 6. Control pH system, buffer change: Nominal controller (-), conservative controller (- - ), and setpoint $(\cdots)$.

Model Approaches to Modelling and Control (R. Murray-Smith and T. A. Johansen, Eds.). Taylor \& Francis Inc.. Bristol, PA. pp. 3-72.

Morari, M. and E. Zafiriou (1989). Robust Process Control. Prentice-Hall. Englewood Cliffs, NJ.

Narendra, K. S. and J. Balakrishnan (1997). Adaptive control using multiple models. IEEE Trans. on Automat. Contr. 42(2), 171187.

Schott, K. D. and B. W. Bequette (1997). Multiple model adaptive control. In: Multiple Model Approaches to Modelling and Control (R. Murray-Smith and T. A. Johansen, Eds.). Taylor \& Francis Inc.. Bristol, PA. pp. 269291.

Wojsznis, W. K. and T. L. Blevins (2002). Evaluating PID adaptive techniques for industrial implementation. In: Proc. of the 2002 Amer. Contr. Conf.. pp. 1151-1155. 

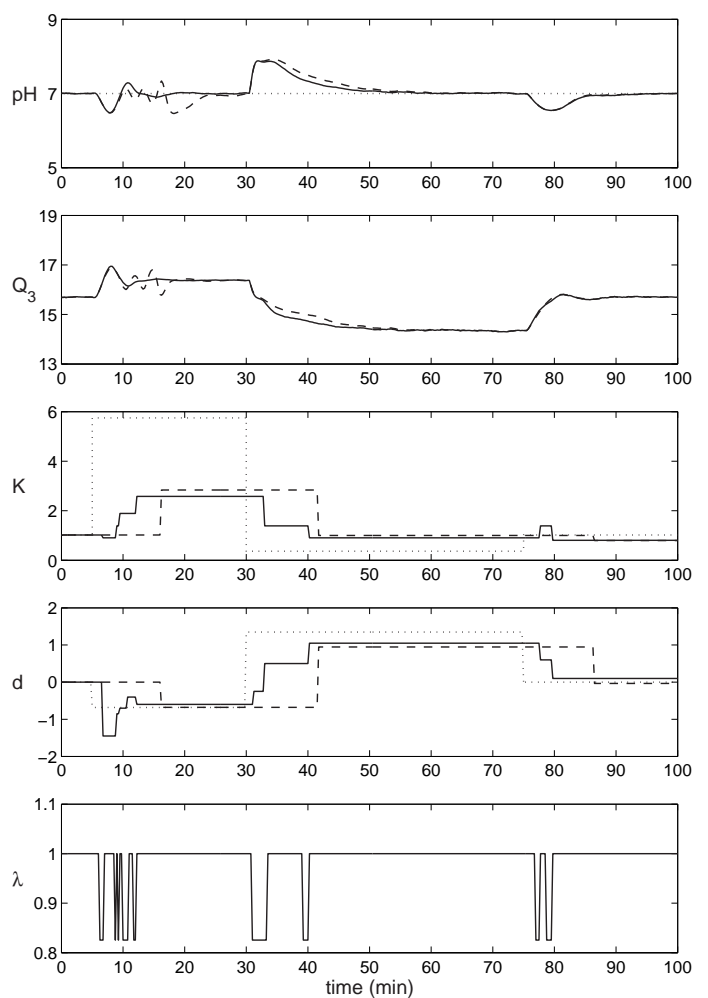

Fig. 7. Control of $\mathrm{pH}$ system, buffer change: Switching controller (-), re-tuning controller $(---)$, setpoint and true values $(\cdots)$. Prediction error used in forgetting.
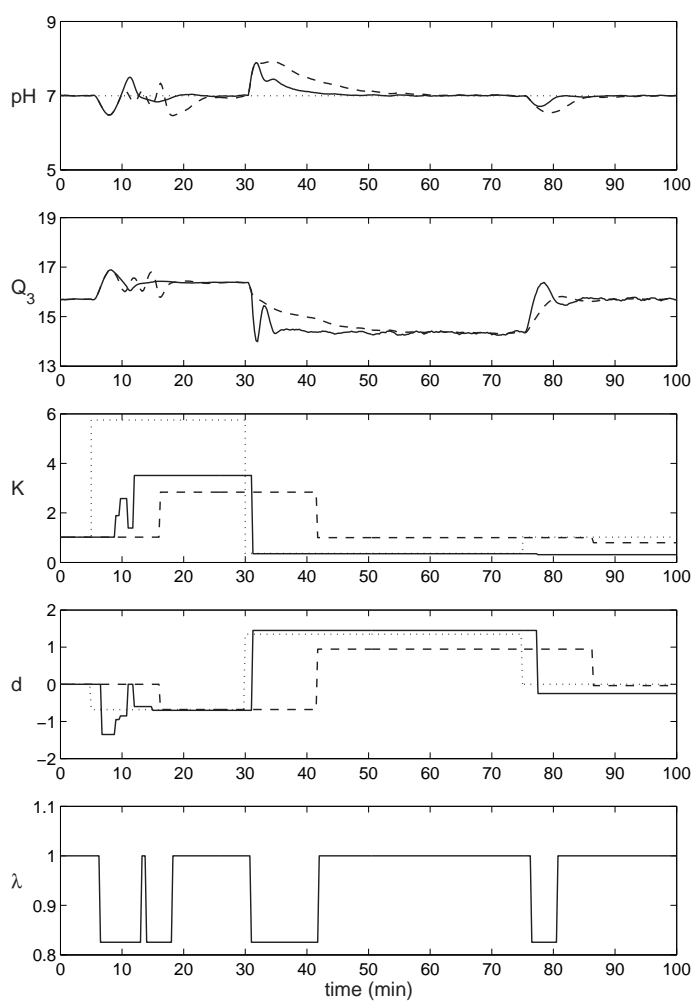

Fig. 8. Control of $\mathrm{pH}$ system, buffer change: Switching controller $(-)$, re-tuning controller $(--)$, setpoint and true values $(\cdots)$. Control error used in forgetting.
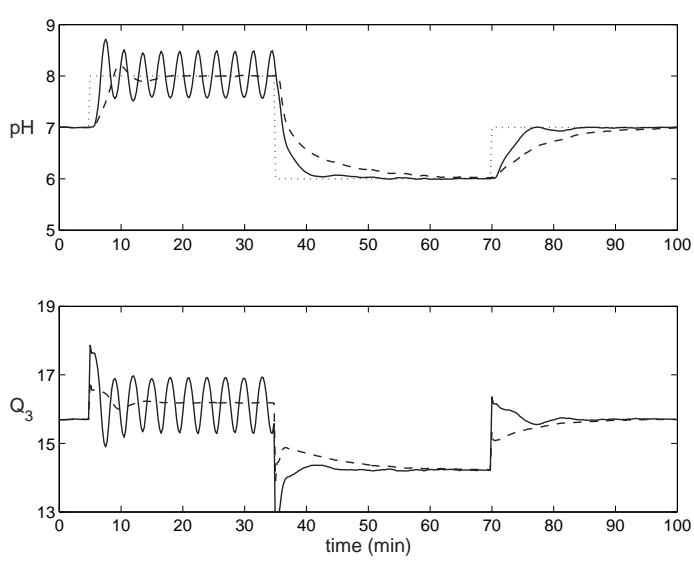

Fig. 9. Control of $\mathrm{pH}$ system, setpoint change: Nominal controller (-), conservative controller (- - ), and setpoint ( $\cdots)$.
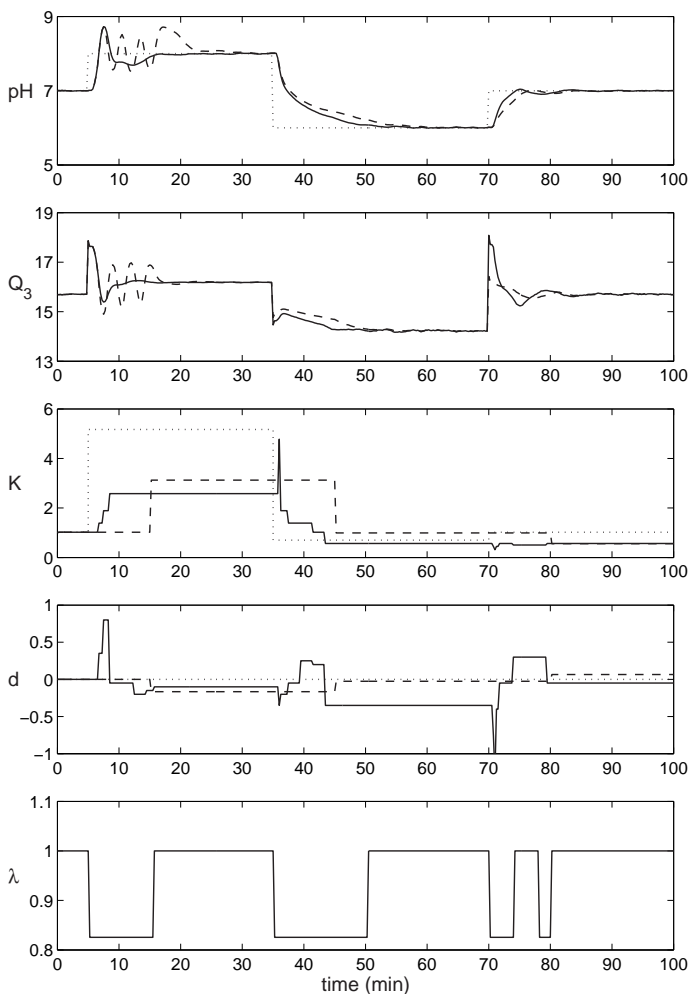

Fig. 10. Control of $\mathrm{pH}$ system, setpoint change: Switching controller (-), re-tuning controller $(---)$, setpoint and true values $(\cdots)$. Control error used in forgetting. 